\title{
Molecular and phenotypic evaluation of a novel germline TMEM127 mutation with an uncommon clinical presentation
}

\section{Yilun Deng1, Shahida K Flores', ZiMing Cheng', Yuejuan Qin', Robin C Schwartz²,3, Carl Malchoff³ and Patricia L M Dahia'}

'Division of Hematology and Medical Oncology, Department of Medicine, Cancer Therapy and Research Center, University of Texas Health Science Center at San Antonio (UTHSCSA), San Antonio, Texas, USA

2Department of Genetics and Genome Sciences, UCONN Health, Farmington, Connecticut, USA

3University of Connecticut Carole and Ray Neag Comprehensive Cancer Center, Farmington, Connecticut, USA

Correspondence should be addressed to P L M Dahia: dahia@uthscsa.edu

The authors and journal apologise for an error in the above paper, which appeared in volume 24 part 11, pages L79-L82. The error relates to the Funding section on page L82:

The original text stated:

'This work was supported by the Cancer Prevention and Research Institute of Texas (CPRIT) Individual Investigator Grants RP101202 and RP57154 ...'

This should have stated:

'This work was supported by the Cancer Prevention and Research Institute of Texas (CPRIT) Individual Investigator Grants RP101202 and RP140473 ...' 